\title{
ALGUNAS PRECISIONES EPIGRÁFICAS SOBRE LA ESTELA DE GUALDA (CIFUENTES, GUADALAJARA)
}

\author{
POR \\ ARMIN U. STYLOW \\ Centro CIL II, Universidad de Alcalá *
}

PALABRAS CLAVE: Epigrafía latina. Estelas funerarias. Inscripciones reutilizadas. Poblado visigodo de El Tesoro de Carramantiel (Gualda, Guadalajara). Organizaciones suprafamiliares. Turocom. Siglos I d.C.

KEY WORDS: Latin epigraphy. Roman tombstone relief Reused inscriptions. Visigothic village of El Tesoro de Carramantiel (Gualda, Guadalajara). Local communities. Turocom. $1^{\text {st }}$ century AD

\section{RESUMEN}

Un estudio pormenorizado de la estela bisoma de Gualda permite explicar su forma singular y presentar una nueva lectura de sus inscripciones.

\section{SUMMARY}

A detailed study of the Gualda stele provides an explanation of its unusual shape and a revised version of its inscriptions.

La estela de la que trataremos a continuación fue hallada en 1995, en el curso de las excavaciones efectuadas en el poblado hispano-visigodo de El Tesoro-Carramantiel, situado a $1 \mathrm{~km}$ al suroeste del pueblo alcarreño de Gualda, una pedanía de Cifuentes enclavada cerca de la cabecera del embalse de Entrepeñas, y fue dada a conocer dos años más tarde ${ }^{1}$. Hoy se encuentra depositada en el Centro Cultural «Santo Domingo», de Cifuentes, donde la hemos podido estudiar en dos ocasiones ${ }^{2}$. La estela, labrada en una piedra caliza gris clara, algo rojiza, presenta una forma que, a primera vista, resulta algo desconcertante: es un paralelepípedo de $83 \mathrm{~cm}$ de

${ }^{*}$ Este trabajo ha sido facilitado por una ayuda concedida al Centro CIL II por la Obra Social y Cultural de IberCaja.

1 M. A. Cuadrado Prieto-M. Vallejo Girvés, «Hallazgo de una inscripción dual latina en el hábitat hispano-visigodo de Gualda (Guadalajara)», Kalathos 16, 1997, 129-135, con fotografía. Cf., con nuestra lectura corregida, M. E. Ramírez Sánchez, Epigrafía y organización social en la región celti bérica: los grupos de parentesco, tesis doctoral leída en 1999 en la Universidad de Las Palmas de Gran Canaria (en trance de publicación), Catálogo A.66. Agradecemos al autor el haber podido discutir con él ese nuevo testimonio.

2 Agradecemos al excavador del yacimiento, D. Migue Ángel Cuadrado Prieto, sus valiosas indicaciones acerca de las circunstancias del hallazgo de la estela y al personal del Centro Cultural las facilidades ofrecidas para su estudio. Las fotografías fueron tomadas por $\mathrm{H}$. Gimeno Pascual alto, $68 \mathrm{~cm}$ de ancho y $27-30 \mathrm{~cm}$ de grueso, que ha sido someramente desbastado en la parte trasera mientras que está alisado en los costados y en la cara frontal, donde presenta dos campos epigráficos ligeramente rebajados, de 30 por $25 \mathrm{~cm}$ cada uno, separados por un delgado baquetón vertical. Ambas esquinas superiores han sido redondeadas para formar un remate aproximadamente semicircular (que, sin embargo, no alcanza la parte central, horizontal), pero ese redondeado se ha ejecutado solamente en la mitad trasera del grueso de la estela, conservándose en la mitad anterior del grueso las esquinas antiguas, si bien disminuidas: la derecha, por los desconchones que ha sufrido con posterioridad, y, la izquierda, por un corte aproximadamente rectangular, consecuencia quizás de la reutilización de la estela como material constructivo en una de las casas del poblado.

Con todo, es perfectamente reconocible la forma primitiva de la estela, que corresponde a un tipo bien conocido por un gran número de ejemplos en muchas partes - sobre todo en el Sur y el Centrode la Península: ante un fondo rectangular, que queda en segundo plano, destaca un remate semicircular conseguido por medio del rebaje redondeado de las esquinas superiores en la parte frontal del grueso del cuerpo. Sin embargo, las estelas de ese tipo llevan la inscripción sin excepción en la cara adornada por el remate semicircular, jamás en la cara rectangular, como el presente ejemplar de Gualda. ¿Cómo se explica el tratamiento tan singular en este caso?

Un atento examen de la parte trasera de la estela, con el remate semicircular, nos revela que la singularidad es sólo aparente y que era en ella donde, en un principio, se iba a grabar el epígrafe. Aquí observamos que se había empezado a practicar un rebaje para el campo epigráfico (fig. 1; las huellas más claras aparecen en el lado izquierdo), y que pronto se desistió de tal trabajo, posiblemente porque apareciera, en plena zona prevista para la inscripción, uno de esos huecos producidos por la erosión que a menudo desfiguran ese tipo de piedra caliza (y que, de menores dimensiones, también se ven por doquier en la parte inscrita). Un indicio de 


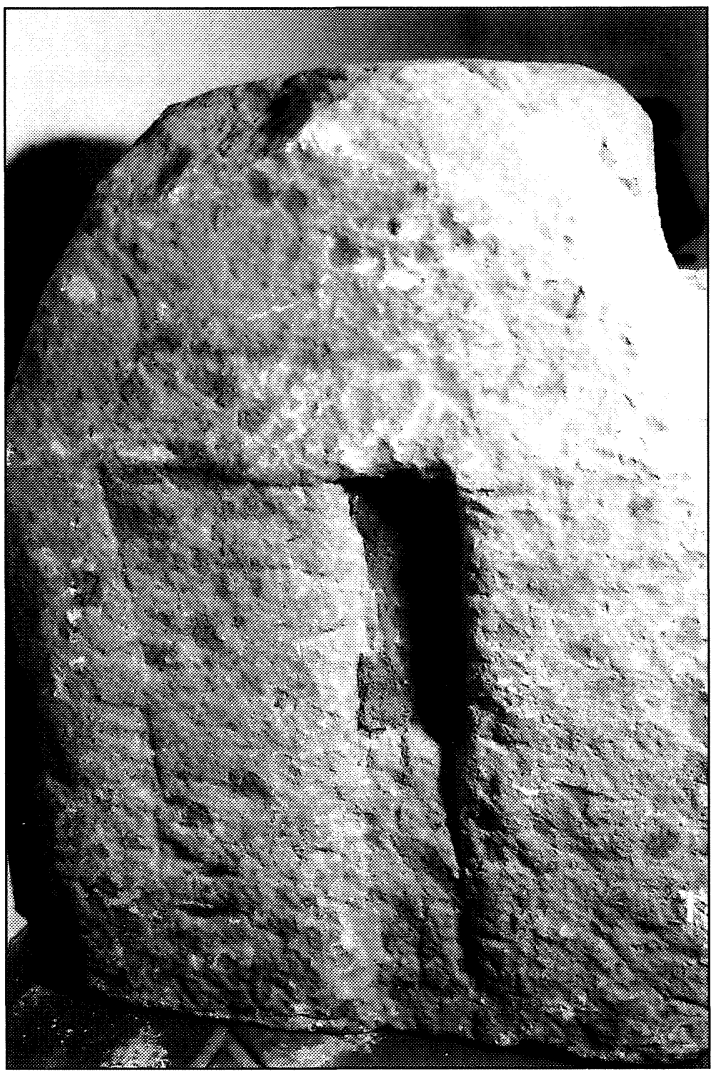

Fig. 1.-Parte trasera de la estela de Gualda.

que hubiesen topado con un desperfecto de esa índole pudiera ser el canalillo vertical, de distinta profundidad, que, por mor de cata, se grabó en el centro del área trazada y que no creemos, como sospechan los editores ${ }^{3}$, hubiera servido para engarzar algún tipo de material constructivo en el momento de la reutilización de la estela y menos para que fuera colgada en su primitivo emplazamiento. Más bien parece que, ante la perspectiva de tener que rebajar en varios centímetros todo el campo, optaron por dar la vuelta a la estela y utilizar como cara frontal la parte originariamente trasera, que entonces recibiría los dos campos rebajados para la inscripción y sería alisada, junto con los costados.

La doble inscripción está grabada con caracteres capitales relativamente bien formados y -pese a los mencionados desperfectos de la piedra y los efectos de la erosión- no presenta mayores problemas de lectura. Existe un claro, aunque no totalmente logrado, intento de paginación: Incluso la oscilación en la altura de las letras que se observa (lado $a$, ll. 1 y $3: 4,5 \mathrm{~cm}$; 11.2 y $4: 4 \mathrm{~cm}$; $1.5: 3,5 \mathrm{~cm}$; lado $b, 1$.

${ }^{3}$ Cuadrado-Vallejo, cit. (n. 1), 130.
1: 4-4,5 cm; 1. 2: 3,5 cm; 1l. 3 y 4: 4-5 cm; $1.5: 4,5$ $\mathrm{cm}$; las letras redondas como $\mathrm{C}, \mathrm{G}$ y $\mathrm{O}$ generalmente son más pequeñas, como es habitual en las inscripciones latinas, aunque aquí la diferencia es más marcada) parece ser intencionada, obedeciendo quizás a un deseo de destacar las distintas unidades lógicas. Se evitan en lo posible las separaciones de palabras (sólo en el lado a, ll. 3/4), y las letras se espacian más o menos para llenar por completo los renglones, alineados por la izquierda. Los pequeños signos de interpunción parece que son triangulares aunque la erosión no permite pronunciarnos al respecto con seguridad. Los textos rezan (fig. 2):

$\begin{array}{ll}\text { Inscripción } a: & \text { Inscripción } b: \\ & \text { Gaius } \\ \text { Atta } & \text { Turoco }(m) \\ \text { Turoco }(m) & \text { Pacati } \\ \text { Gai } u- & f(\text { ilius }) \cdot v i v(u) s \cdot \\ \text { xsor } & (\text { ibi }) \cdot \text { m }(\text { onumentum }) \\ h(\text { ic }) \cdot s(\text { ita }) \cdot e(\text { st }) \cdot s(\text { it }) & \\ t(\text { ibi }) \cdot t(\text { erra }) \cdot l(\text { evis }) & f(\text { aciendum }) \cdot c(\text { uravit })\end{array}$

Nuestra lectura coincide en gran parte con la que daban los primeros editores, quienes, sin embargo, no apreciaban la interpunción, entendían Turtoqo(n) (con nexo RT) en 1. a 2 (y, por consecuente, restituían $T u[r t]$ oqo( $n)$ en $1 . b 2$ ), leían en $1 . b 3$ solamente $P a[.] t$.$i y daban un vacat al comienzo de 1 . b$ 5 en lugar de la letra $S$, de la que sólo se conserva el arranque superior, pero cuya existencia es apoyada no sólamente por el sentido de la fórmula, sino también por la ordinatio del texto y la interpunción que sigue. Posiblemente hay interpunción también entre I y V en 1. a 3.

Ambas inscripciones parece que se grabaron en un mismo momento, a juzgar no sólo por las semejanzas paleográficas ${ }^{4}$, sino también por el contenido de los epígrafes: la estela se puso después del fallecimiento de Atta, la esposa de Gaius, el cual es designado sólo indirectamente como el autor de la inscripción conmemorativa de su mujer (el gamónimo Gai uxsor que figura en el lugar habitual del patronímico de la esposa sustituye en cierto sentido a la fórmula uxori et sibi que cabría esperar en la inscripción del marido), ya que la segunda inscripción sólo expresa la voluntad del marido de que bajo ese monumento que erigió en vida deben ser sepultados también sus propios restos mortales. Sorprende la soltura con la que se utilizan las fórmulas sepulcrales y dedicatorias, casi todas abreviadas y en parte

${ }^{4}$ Nótense, p. ej., los característicos bucles inferiores casi angulados de las S. Formas notables son además la $\mathrm{P}$ muy abierta en $1 . b 3$ y la $\mathrm{M}$, cuyo ángulo central no llega hasta la línea base, en $1 . b 5$. 
sin paralelos en la Carpetania, caso de $s(i b i) \operatorname{viv}(u) s$ y $m($ onumentum $)^{5}$, considerando el indigenismo estructural de la onomástica de los dos personajes: ambos son designados por un nombre individual -indígena en el caso de la esposa ${ }^{6}$, mientras que el marido utiliza como nombre propio el praenomen latino Gaius ${ }^{7}$ - , seguido por el genitivo de plural de una unidad organizativa indígena, $\operatorname{Turoco}(m)$. La fórmula onomástica de la esposa se completa por el gamónimo que aquí, probablemente por la razón que acabamos de comentar, sustituye al habitual patronímico, mientras que el marido lleva un

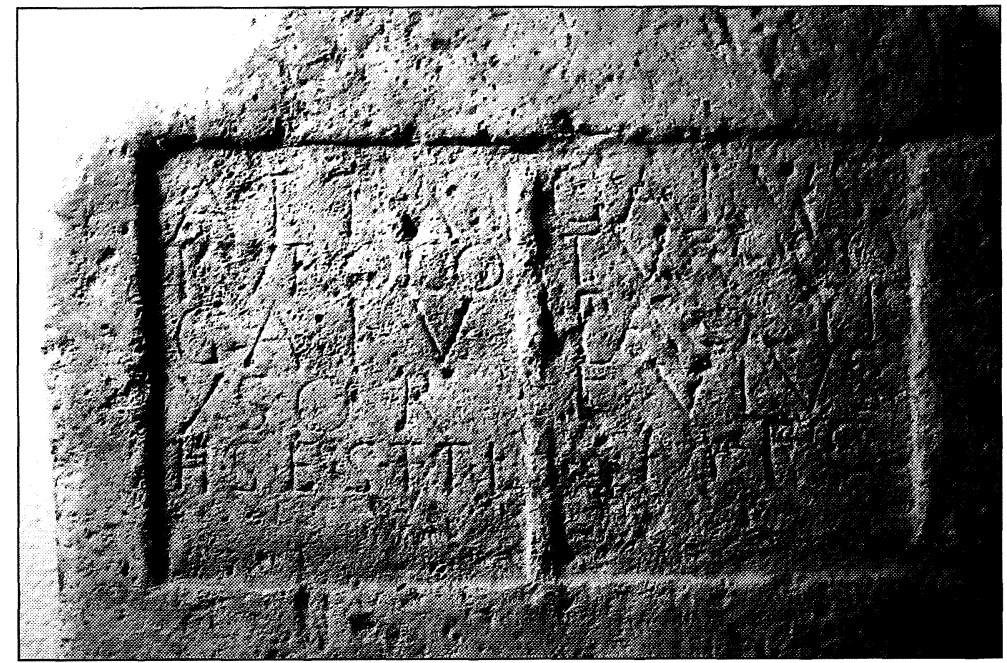

Fig. 2.-Inscripción de la estela de Gualda. patronímico latino no significativo ${ }^{8}$, ampliado por f(ilius).

El nombre de la unidad organizativa indígena es nuevo, apareciendo, como ocurre en la gran mayoría de los casos, sólo en esta inscripción. El radical Tur-, sin embargo, del que está derivado, es frecuente en la onomástica celtibérica y ha dado, entre otros, los bien conocidos antropónimos indígenas

5 Aunque la abreviatura m(onumentum) (naturalmente fuera de la fórmula $h(o c)$ m(onumentum) $h$ (eredem) $n($ on) $s$ (equetur) ) es particularmente frecuente en las zonas colindantes con la Cordillera Cantábrica, no faltan ejemplos más cercanos al Sistema Central, como, p. ej., en la provincia de Soria (Cuevas de Soria: CIL II 2843 con la resolución propuesta por M. C. González Rodríguez, Las unidades organizativas indígenas del área indoeuropea de Hispania, Vitoria 1986, 130; Muro de Agreda: A. Jimeno, Epigrafía romana de la provincia de Soria, Soria 1980, 76; Santervás de la Sierra: Jimeno, ibid., 107). En la misma zona un ejemplo de $v$ (iva) $s($ ibi) ...f(aciendum) $c$ (uravit): Jimeno, ibid., 71 (Medinaceli).

6 Para Atta, repartido por toda la Meseta Norte y las zonas colindantes de la Meseta Sur, cf. M. L. Albertos Firmat, La onomástica personal primitiva de Hispania: Tarraconense y Bética, Salamanca 1966, 42, con las correcciones y puesta al día de J. M. Abascal Palazón, Los nombres personales en las inscripciones latinas de Hispania, Murcia 1994, 289-290.

7 Al contrario de lo que ocurre con el uso relativamente frecuente de Lucius o Marcus como nombre individual (cf. Abascal, cit. n. 6, 404 y 414, respectivamente), parece que sólo hay otro testimonio hispánico más para Gaius, un grafito inédito sobre terra sigillata hispánica, procedente de Complutum (T. E. A. R., inv. n. ${ }^{\circ} 5 / 1 / 2 / 96 / 1759$ ). Para su uso como cognomen véase O. Salomies, Die römischen Vornamen. Studien zur römischen Namengebung, Helsinki 1987, 165.

${ }^{8}$ Para Pacatus cf. I. Kajanto, The Latin cognomina, Helsinki 1965, 261; en Hispania: Abascal, cit. n. 6, 446 (repartido sobre todo en las zonas más romanizadas, aunque no faltan algunos ejemplos del área indoeuropea, p. ej., CIL ·I 923, de Talavera de la Reina, CIL II 2636, de La Milla del Río, León, o HAE 1159, de Idanha-a-Velha).
turos/Turos en lengua celtibérica ${ }^{9}$ y Tur(e)ius en la correspondiente forma latina ${ }^{10}$. El nombre de la unidad organizativa está formado con el sufijo -oco-, el segundo más frecuente (después de $-i c o-{ }^{11}$ ) en tales formaciones, aunque es el primer ejemplo de ese sufijo con la variante -om/-on en lugar de la más común -um/-un. Además, es preciso destacar que nuestra inscripción es el segundo testimonio de un matrimonio que comparte un mismo nombre de unidad organizativa indígena ${ }^{12}$, si bien todavía no sabemos si ello debe interpretarse como el reflejo de la situación originaria de los dos individuos, es decir, si se trata de un matrimonio entre parientes relativamente próximos, o como indicio de que uno de los cónyuges con su matrimonio hubiera pasado a integrarse en la unidad organizativa del otro ${ }^{13}$.

${ }^{9}$ En grafía celtibérica en el Bronce de Botorrita III (F. Beltrán Lloris-J. de Hoz-J. Untermann, El tercer bronce de Botorrita (Contrebia Belaisca), Zaragoza 1996, col. I 1. 2, y varias veces más en el genitivo turo) y en grafía latina en Peñalba de Villastar (J. Untermann, Monumenta Linguarum Hispanicarum, IV: Die tartessischen, keltiberischen und lusitanischen Inschriften, Wiesbaden 1997, 632-633, K.3.11, en genitivo ibid., K.3.12).

10 Repartidos sobre todo en las zonas vetona y lusitana: HAE 1051. 1309 (Lumbrales, Salamanca); CIL II 947=5344 = HEp 3, 1993, 377 (Torralba de Oropesa, Toledo); J. d'Encarnação, Divindades indigenas sob o dominio romano en Portugal. Subsidios para o seu estudio, Lisboa 1975, 257 (Fail, Viseu); HAE 2328 (Viseu); CIL II 744.745 (Brozas, Cáceres); CIL II 788 (Coria, Cáceres); AE 1977, 396 (Truji1lo, Cáceres); AE 1983, 483 (Almacave, Lamego, Viseu); AE 1979, 328 (Baraçal, Sabugal, Guarda):

11 Efectivamente aparece turikum en el Bronce de Botorrita III (col. III 1. 4).

12 El otro caso es AE 1925, 22, de Barcebalejo (Soria).

13 Posibilidad ésta menos probable en la situación actual de nuestros conocimientos, puesto que existen casos donde 
Para la datación de la estela, sus primeros editores proponían una fecha comprendida «entre la segunda mitad del siglo I d. C. y la primera mitad del siglo II d. C.» ${ }^{14}$, pero no aducían argumentos solventes para ella ${ }^{15}$. Mientras que en la onomástica se constata, por un lado, la adopción parcial y más bien superficial de modelos $-\mathrm{y}$ de nombres- romanos, conservándose, por otro, las costumbres onomásticas indígenas, las fórmulas utilizadas, sus abreviaturas y grafías (p. ej., uxsor por uxor) denotan, al contrario, una notable familiaridad con los hábitos epigráficos romanos. La fórmula sit tibi terra levis, que en Hispania aparece a partir de época tiberiana ${ }^{16}$, proporciona un terminus ad quem o post quem, que, en vista de la forma marcadamente antigua de la $\mathrm{P}$ abierta en $b$ 1. 3 y de la ausencia de la indicación de la edad de la esposa difunta, difícilmente cabe rebasar mucho. Por ello proponemos una datación de la estela en época julio-claudia no muy avanzada, anterior pues a la mitad del siglo I.

Por la situación en que se localizó lạ estela —reutilizada (boca abajo) como sillar en la junta de dos paredes del poblado hispano-visigodo de El Tesoro-Carramantiel, fechado en el siglo viI por los ajuares encontrados en las tumbas de la necrópolis asociada a él ${ }^{17}$ — así como por la ausencia de cualquier rastro de hábitat romano en las inmediaciones de dicho poblado llegaron ya los primeros editores a la conclusión - acertada, sin lugar a dudas - de que la estela había sido trasladada desde su emplazamiento primitivo por los habitantes del poblado en el siglo vII, barajando como lugar de procedencia más probable la villa romana de Gárgoles de Arriba, situada aguas arriba del Río Cifuentes, a unos 8 $\mathrm{km}$ del poblado ${ }^{18}$, sin excluir otros yacimientos ro-

marido y mujer pertenecen a distintos grupos de parentesco (CIL II 5789. 6294). Cf. la discusión del tema en Ramírez Sánchez, cit. (n. 1), cap. 2.5.1. Agradecemos vivamente al autor el permiso de utilizar su estudio.

${ }^{14}$ Cuadrado -Vallejo, cit. (n. 1), 131

15 Los «criterios internos» aducidos se limitan a la onomástica mixta, no distinguiéndose, en el caso de la esposa, entre los antropónimos indígenas Atta/Atto y el gentilicio latino Attius y, en el del marido, entre el uso banal del praenomen Gaius en una onomástica al estilo romano (el caso citado es el de un liberto, CIL II 6308) y su utilización, como en la presente inscripción, como nombre individual.

${ }^{16} \mathrm{Cf}$. CIL II $\mathrm{I}^{2} / 7,441$; A. U. Stylow, «The beginnings of Latin epigraphy in Baetica: the case of the funerary inscriptions», en The archaeology of early Roman Baetica (ed. S. Keay, Journal of Roman Archaeology, Suppl. Series 29), Portsmouth/Rhode Island 1998, 114 nota 36.

17 A falta de la publicación de los resultados de la excavación remitimos a los datos aportados por M. A. Cuadrado Prieto y M. Vallejo Girvés en su edición, cit. (n. 1), 129. 134.

18 Para ella, véase J. Sánchez-Lafuente, «La epigrafía y el entorno arqueológico de la villa romana de Gárgoles de Arriba (Guadalajara)», Lucentum 6, 1987, 175-183. manos enclavados ya en las proximidades del cauce del Tajo, como los de Trillo y de Carrascosa de Tajo ${ }^{19}$.

Se puede aportar otro argumento más que redunda en esa idea de que la estela ha sido acarreada desde otro sitio, y es precisamente su material lítico, una caliza dura y compacta, mientras que para todos los muros del poblado y para las tapas de las tumbas de la necrópolis se ha utilizado la arenisca local sobre la que se asientan tanto el poblado hispano-visigodo como el próximo pueblo de Gualda. El material de la estela es asimismo un argumento en contra de su supuesta procedencia de la villa romana de Gárgoles de Arriba, porque las diversas estelas sepulcrales romanas halladas allí no sólo salieron o bien descontextualizadas o bien reutilizadas como material de construcción, lo que arroja ciertas dudas sobre su pertenencia a dicha villa, sino, sobre todo, porque su material — donde hay constancia de él- es piedra arenisca ${ }^{20}$. La procedencia exacta de la estela de Gualda sigue siendo pues una incógnita, aunque probablemente provenga de una zona del valle alto del Río Cifuentes, aguas arriba de Gárgoles, donde sí aflora la caliza, y con seguridad del entorno general de esa primera llanura que abre el río Tajo tras abandonar el curso alto de su nacimiento, llanura que en la Antigüedad probablemente pertenecía al territorio del municipio de Ercávica ${ }^{21}$ y era cruzada por la calzada romana de Segontia a Segóbriga ${ }^{22}$.

Con todo, queda claro que la estela fue transportada desde una considerable distancia, y no se entiende bien la finalidad de tanto esfuerzo - sólo la subida de la pieza, que pesa media tonelada aproximadamente, al lugar de su reutilización, por una cuesta difícilmente practicable con carros, constituye toda una proeza-, considerando que lo que no falta en aquel enclave es precisamente la piedra; la arenisca local es perfectamente idónea para la construcción, como lo demuestran los mismos muros de muy buena sillería del poblado hispano-visigodo.

19 Para ellos, cf. Sánchez-Lafuente, cit. (n.18), 180-181; J. M. Abascal Palazón-J. R. López de los Mozos, «Dos inscripciones inéditas de los territoria de Segontia y Ercavica», HAnt 17, 1993, 275-277.

${ }^{20}$ Sánchez-Lafuente, cit. (n. 18), 177 ss. El único caso dudoso es su núm. 3 (p. 177), que, según él, sería de piedra caliza, mientras que G. Alföldy (ficha CIL II, autopsia) la define como arenisca.

${ }^{21}$ En este sentido se expresan, aunque con dudas, también Abascal-López, cit. (n. 19) , 276.

22 J. M. Abascal Palazón, Vías de comunicación romanas de la provincia de Guadalajara, Guadalajara 1982, 89 ss. 